\title{
Dimensional Evaluation of Soft Tissue Changes Following Mandibular Advancement And Setback Orthognathic Surgeries- A Systematic Review
}

\author{
Janani Ravi', Janani Jayapal'², S.Dilip ${ }^{3}$ \\ ${ }^{1}$ Senior Lecturer, SRM Dental College, Chennai, Tamil Nadu, India, ${ }^{2}$ Senior Lecturer, Meenakshi Ammal Dental \\ College, Chennai, Tamil Nadu, India, ${ }^{3}$ Professor, Department of Orthodontics And Dentofacial Orthopaedics, Srm \\ Dental College, Chennai, Tamil Nadu, India
}

Background: The principle goal of orthognathic surgery is to establish a balanced and stable dento-skeletal facial complex. This mandates the surgeon and the orthodontist to be able to predict the soft tissue changes to the orthognathic surgery precisely, which is accurately possible using 3-D imaging.

Aims: To evaluate the soft tissue changes following mandibular advancement and mandibular setback orthognathic surgeries using 3-D imaging.

Methods and Material: This review was conducted according to Preferred Reporting Items for Systematic Reviews and meta-Analyses guidelines systematically searching the six databases including PubMed, Cochrane, Google Scholar, LILACS, Directory of Open Access Journals, and OpenGrey.

Conclusion: In mandibular advancement surgeries, the hard to soft tissue movement ratio was 1:0.97. There is an increase in volume of both lip area and chin. In mandibular setback, there was increased gradient of change from upper lip and lower lip to the chin. It was also seen to extend from the midline ( stomion ) to the lateral ( Chelion). The ratio of the soft tissue to bony movement was about $66 \%$ at the Li point.

Keywords : Mandibular advancement, Mandibular setback, 3-D imaging, CBCT and Facial soft tissue changes

\section{Introduction}

Treatment of severe skeletal malocclusion frequently requires an orthognathic surgical approach involving either mandibular advancement or setback to improve facial esthetics and to harmonize the facial profile. The ultimate goal of treatment is to achieve a balanced soft tissue profile ( Ackerman JL 1999 , Park YC et al 1986) ${ }^{1,18}$. Many studies have attempted to predict the behaviour of soft tissues after surgery ( Bailey LJ et al 1996, Bell WH et al 1986) ${ }^{4,5}$.

\section{Corresponding author:}

\section{Dr. Janani Ravi}

Senior Lecturer, SRM Dental College, Chennai, Tamil Nadu, India, ${ }^{2}$ TELEPHONE : 8754603287

Email: janani23294@gmail.com
The changes in the relationship of hard tissue to soft tissue were first reported by McNeill et al in 1972.( Landes CA et al 2002) ${ }^{13}$. Since then, several investigators have addressed their attention on soft tissue responses following mandibular advancement and setback. The past research paper involving the soft tissue changes had the short comings of being observational period involving a maximum a 3 year period and a particular study by Eggensperger et al for a period of 12 years ( Eggensperger et al 2007) $)^{7}$. The main drawback of these studies have been the 2-Dimensional nature ( Incrapera AK et al 2010, Khanehamasjedi M et al 2010). ${ }^{9,11}$ These limitations channeled the present studies into more accurate platform involving 3-dimensional approaches.

This current systematic review is an attempt to bridge the gap between the soft tissue outcomes of orthognathic surgery involving either mandibular 
advancement or setback in a 3-Dimensional imaging system. Thus, the current study evaluates the soft tissue changes in patients who have undergone mandibular advancement or mandibular set back surgery evaluated using 3D imaging techniques.

\section{Materials and Methods}

\section{Protocol and Registration}

This review was based on a specific protocol developed and piloted following the guidelines outlined in the Preferred Reporting Items for Systematic Reviews and Meta-Analyses (PRISMA) P statement ( Shamseer L et al 2015, Higgins JP et al 2011, Liberati A et al 2009 ) $19,8,14$

\section{Eligibility Criteria}

Eligibility criteria was based on the research question defined in the PICO format. Do patients who have undergone mandibular setback or mandibular advancement $(\mathrm{P})$ evaluated using 3D imaging techniques (I) exhibit before and after (C) changes in the facial soft tissues $(\mathrm{O})$.

The inclusion criteria primarily composed of human clinical trials, patients of either sex who were over the age of 18 years, who had undergone single jaw mandibular orthognathic surgeries for class II or class III correction, had 3-D records, Data published in 20092019 which have been published in English.

The exclusion criteria included animal or in vitro studies, narrative or literature reviews, and case reports and series, patients who have cleft lip and palate, craniofacial disorders, degenerative conditions, trauma, temporomandibular joint pain, previous orthognathic surgery, inflammatory conditions, degenerative conditions, facial asymmetries.

\section{Search strategy for identification of studies}

The search was carried out in two parts. The first is an electronic search done based on a search strategy developed on PICO format and was checked using the PRESS checklist for systemic reviews. The search terms includes controlled vocabulary, author keywords, boolean operators and truncations was appropriately used and revised for each data base, considering the differences in controlled vocabulary and syntax rules. The following electronic databases were searched: PubMed, Google scholar, LILIACS, Cochrane registry of clinical trials, DOAJ, and unpublished literature was searched on opengrey.eu. The second part of the search was a hand-search of the relevant journals to the systemic reviews was done. The following journals were searched:

-American journal of orthodontics and dentofacial orthopaedics

-British journal of orthodontics

-European journal of orthodontics

-Journal of Indian orthodontic society

-Korean journal of orthodontics

-The Angle orthodontist

-World journal of orthodontics

-Journal of Cranio-Maxillo-Facial Surgery

-International Journal Oral and Maxillofacial Surgery

- American association of oral and maxillofacial surgeons

- The hand search included screening of contents, title and abstracts done to identify all relevant studies in the above mentioned journals from January, 2009 to December, 2019. The reference lists of all eligible studies were hand searched for additional studies.

\section{Study selection}

After the duplicate references were removed using references software (MENDELEY 1.19.2, Elsevier, 2018, New york, USA), the studies were assessed for inclusion independently by 2 authors (J.J., J.R) who were not blinded to the identity of the authors of the 
Medico-legal Update, October-December 2021, Vol.21, No. $4 \quad 45$

studies, their institutions, or the results of their research. Study selection procedures comprised reading of titles, abstracts, and full texts. After they excluded non-eligible studies, the full report of publications considered eligible for inclusion by either author was obtained and assessed independently. In case of disagreements between the authors (J.J., J.R) consultations with other author (D.S) was held. A record of all decisions on study identification was kept.

\section{Assessment of Risk of Bias}

Two review authors (J.J and J.R) independently assessed the risk of bias of the eligible trials according to the Cochrane Collaboration's risk of bias tool. In cases of discrepancy, consensus was obtained by consulting a third reviewer (D.S). The domains assessed were (1) random sequence generation; (2) allocation concealment; (3) blinding of participants; (4) blinding of personnel; (5) blinding of outcome assessment; (6) incomplete outcome data; (7) selective reporting; (8) other biases (baseline imbalance, similarity in using cointerventions between groups, and inadequate statistical analysis). The potential risk of bias for each study was classified as high, unclear, or low. (Figure 1, 2)

\section{Data extraction}

Data extraction was performed independently by the same first 2 authors (J.J., J.R). To record the desired information, customized data collection forms were used. Disagreements were resolved in re-evaluations of the original studies by both authors and discussions with the third author (D.S) until consensus was reached.

\section{Results}

The results interpreted in the included articles have been included in the discussion part . Articles comprising of combination surgeries of maxilla along with BSSO advancement or setback have also been chosen but the results interpreted for mandibular surgeries have only been evaluated.

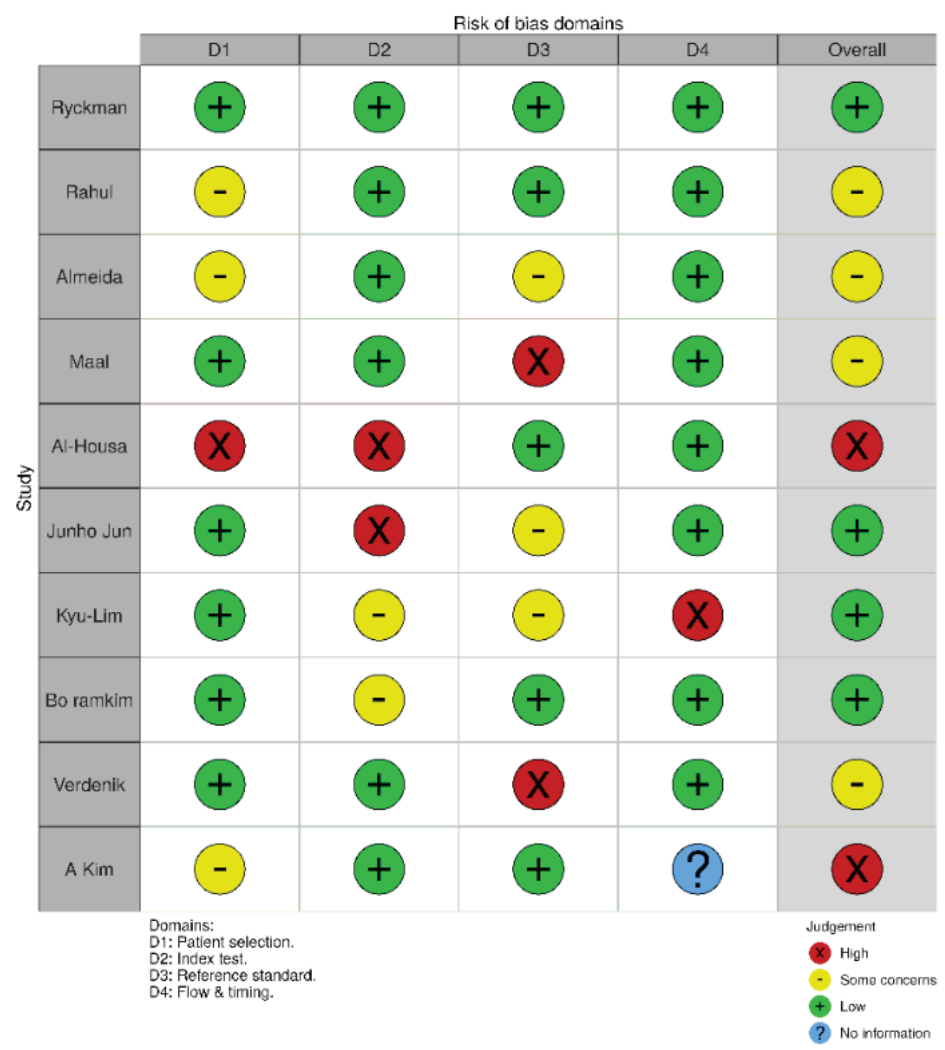

Figure. 1-Risk of bias -Traffic-light plot 


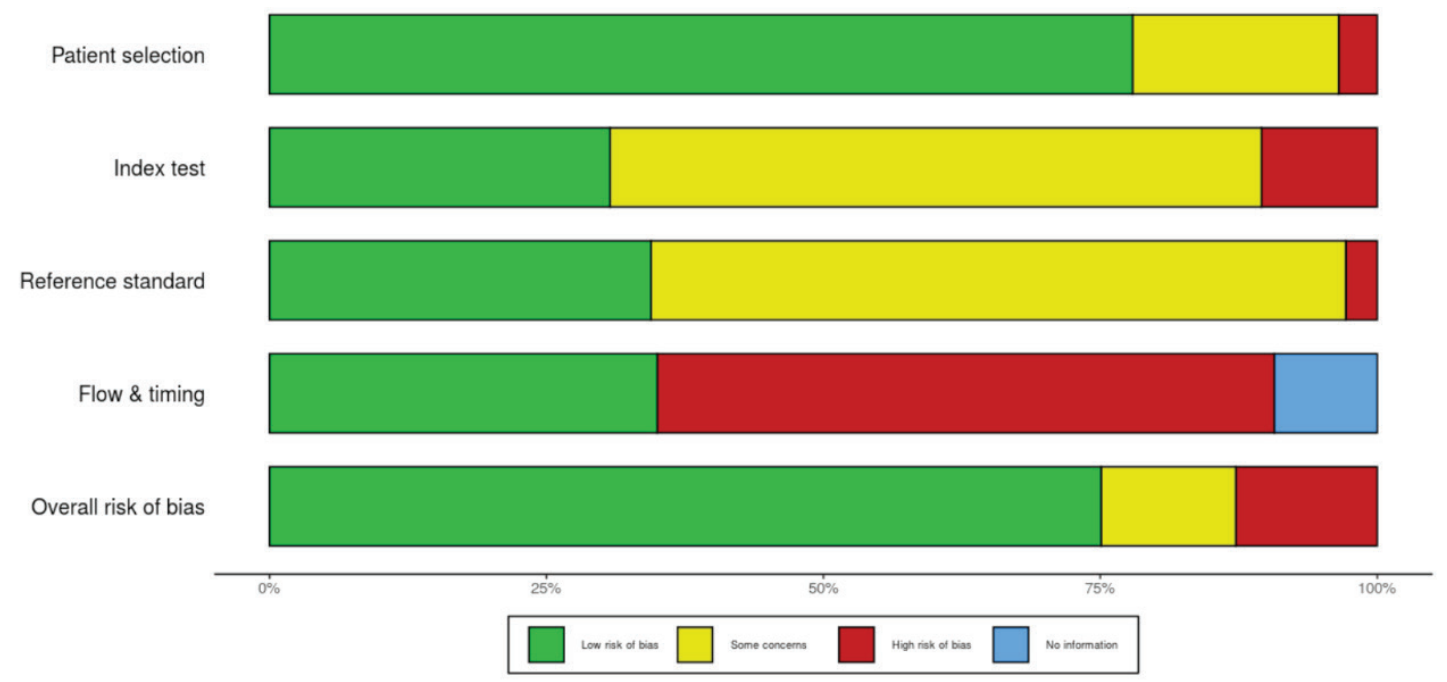

Figure 2. Weighted Summary Plot

\section{Discussion}

Orthognathic surgery involves manipulation of the jaw bones which may have a profound impact on the overlying soft tissues. Facial appearance is an important factor in influencing social and psychological development and plays an crucial role in patients' working status and overall wellbeing ( Rustemeyer $\mathrm{J}$ et al 2012, Modig $\mathrm{M}$ et al 2006 ). ${ }^{21,16}$ So it is of paramount importance to evaluate the changes that can take place in the soft tissues to aid in a proper treatment planning before surgery and to guide the patient and clinicians regarding the esthetic changes that will take place following surgery. Though literature is replete with evidence on the two dimensional evaluation of soft tissue changes after orthognathic surgery, these imaging modalities are often associated with distortion which lacks the precise estimation (Incarpera AK et al 2010, Khanehmasjedi M et al) ${ }^{9,11}$. The purpose of this systematic review was to evaluate the soft tissue changes associated with mandibular setback and advancement surgeries using Cone Beam Computed Tomography.

A total of 10 articles satisfying the inclusion criteria were chosen for the systematic review, ( Ryckman MS et al 2010, Rahul Tiwari P et al 2018, Almeida
RC et al 2011, Maal TJ et al 2012, Al- Housami SA et al 2015, Junho Jung et al 2018, Yong-Kyu Lim et al 2010, Bo- Ram Kim et al 2013, M Verdenik et al 2014 , Kyung-A Kim et al 2019) 22,20,315,2,10,23,6,17,12 out of which 4 articles focused on soft tissue changes following mandibular advancement surgeries, ( Ryckman MS et al 2010, Almeida RC et al 2011, Maal TJ et al 2012, Al Housami SA et al 2015$)^{22,3,15,2} 4$ articles focused on soft tissue changes following mandibular setback surgeries ( Yong-Kyu Lim et al 2010, Bo- Ram Kim et al 2013, M Verdenik et al 2014, Kyung- A Kim et al 2019$)^{23,6,17,12}$ and the remaining 2 articles ( Rahul Tiwari P et al 2018, Yong- Kyu Lim et al 2010) ${ }^{20,23}$ focused on soft tissue changes after orthognathic surgeries for both mandibular deficiencies and excess without any restriction to a particular problem.

The four studies evaluated soft tissue changes following correction of retrognathic mandible based on parameters such as changes in the perioral soft tissues and volumetric changes. Michael S Ryckman et al (2010) concluded that facial soft tissues appear to respond more to anterior movement of the jaws than to an increase in transverse dimensions after comparing the ratio of soft tissue to hard tissue movements in both the dimensions. It was found in his study that mean 
ratios of anteroposterior soft-to-hard tissue movements was $101.1 \% \pm 27.3 \%$ in the sub-commissural region whereas the mean ratios of transverse soft-to-hard tissue movements in the same region was only $82.5 \%$ $\pm 56.7 \%$ ( Ryckman MS et al 2010) ${ }^{22}$. Almeida et al (2011) evaluated the soft tissue response to mandibular advancement using 3D CBCT scanning and concluded that there was a variable soft tissue response after surgery and found upto $73 \%$ changes in the soft tissue chin associated with the movement of the hard chin ${ }^{3}$.

T.J Mall et al (2012) evaluated volumetric changes in the soft tissues using CBCT scan 1 year postoperatively and found a mean volume increase of $4660 \mathrm{~mm}^{3}$ in the region of the chin and a mean volume increase of 1540 $\mathrm{mm}^{3}$ in the lip region. A study conducted by Salah Al-Di Al Housami et al (2015) concluded that the soft tissues follow their underlying skeletal structure in the ratio of 1: 0.97 in the chin area and stressed the importance of changes in the mentolabial fold depth and mentolabial angle following mandibular advancement surgeries ${ }^{15}$

Amongst the 4 studies included in the systematic review for evaluation of soft tissue changes following mandibular setback surgeries, 2 studies evaluated soft tissue changes associated with individual surgeries of Lefort I and BSSO using CBCT scans and combination surgeries of BSSO setback, Lefort I advancement. BoRam Kim et al (2013) concluded that the soft tissue changes in the midfacial area was greater with the two jaw surgery than a single jaw surgery. ${ }^{6}$ Verdenik et al (2014) found that the chin and lower lip soft tissue changes was greatest with that of BSSO surgery and the changes in the soft tissues of the submandibular region was greatest with the combination surgeries of Lefort I advancement and Bilateral Saggital Split Ramus Osteotomy. ${ }^{1}$

Two studies evaluated the soft tissue changes following mandibular setback surgeries alone. YongKyu Lim et al (2010) evaluated the soft tissue changes in the vertical and transverse dimension following mandibular setback surgery and concluded that the three-dimensional soft tissue changes in Class III patients after mandibular setback surgery exhibited increased gradients from upper lip and lower lip to chin as well as from stomion to cheilion. ${ }^{23}$ This study found no significant changes in the transverse dimension. Kyung A Kim et al (2019) concluded that there was significant changes in the transverse dimension as well and the changes were correlative of lip and chin and an increasing gradient of ratios were seen from the lower lip to chin. The anteroposterior dimension saw significant backward positioning of the landmarks from the lower lip to the chin and in the vertical dimension, there was a significant upward movement of the landmarks related to chin. ${ }^{12}$

The findings of two studies that included orthognathic surgeries for both mandibular excess and deficiency as a part of their study sample were included under both Class II and Class III category based on their respective relevance. Junho Jung et al (2018) concluded that the relative ratio of the soft tissue movement to the bony movement after bilateral sagittal split osteotomy was $66 \%$ at the Li point in the anteroposterior direction which was greater than Lefort advancement or setback in which it was $21 \%$ and $14 \%$ respectively. ${ }^{10}$ Thus, it was concluded from this study that soft tissue changes accompanying mandibular movements were greater than maxillary movements.

Rahul Tiwari et al (2018) evaluated soft tissue changes following orthognathic surgical procedures for maxillary and mandibular deformities in all the three planes of space and found that the mentolabial angles significantly increased with mandibular setback procedures $3.27^{\circ}$. Mandibular advancement procedures showed both increase $3.6^{\circ}$ and decrease $7.6^{\circ}$ in the mentolabial angle. ${ }^{20}$

\section{Conclusion}

\section{Mandibular Advancement}

-On assessing all the 3 planes of space, significant changes were encountered in the anteroposterior, transverse and vertical planes.

-Statistically significant difference found for the 
hard to soft tissue movement, 1:0.97 after mandibular advancement.

-Following mandibular advancement, mean volume increase of chin $\left(4660 \mathrm{~mm}^{3}\right)$ and lip area $\left(1540 \mathrm{~mm}^{3}\right)$ was found.

- For every $1 \mathrm{~mm}$ of forward movement of mandible, an increase in the mentolabial angle by $3.6^{\circ}$ was evident.

\section{Mandibular Setback}

-On assessing all the 3 planes of space, significant changes were encountered in the anteroposterior and vertical and some parameters in the transverse plane (lower lip and chin).

- The 3D soft tissue changes in Class III patients after MSS exhibited increased gradients from the upper lip and the lower lip to the chin and as well as from the midline $(\mathrm{Stm})$ to the lateral area $(\mathrm{Ch})$.

- For every $1 \mathrm{~mm}$ of mandibular setback, a decrease in the mentolabial angle by $3.27^{\circ}$

- The relative ratio of the soft tissue movement to the bony movement after bilateral sagittal split osteotomy was about $66 \%$ at the Li point.

Clinical Significance: It is of paramount importance to evaluate the changes that can take place in the soft tissues to aid in a proper treatment planning before surgery and to guide the patient and clinicians regarding the esthetic changes that will take place following surgery

\section{Conflicts of Intrest: Nil}

Source of Funding : Self

Ethical Clearance : Not applicable

\section{References}

1. Ackerman JL, ProffitWR, SarverDM. The emerging soft tissue paradigm in orthodontic diagnosis and treatment planning. Clinical Orthodontics and Research. 1999 May;2(2):49-52

2. Al-Housami SA, Shawky M, El-Morsy K, Abdel-
Ghany H. Three-dimensional soft tissue assessment following mandibular bilateral sagittal split osteotomy. Journal of Craniofacial Surgery. 2015 Nov 1;26(8):e702-6.

3. Almeida RC, Cevidanes LH, Carvalho FA, Motta AT, Almeida MA, Styner M, Turvey T, Proffit WR, Phillips C. Soft tissue response to mandibular advancement using 3D CBCT scanning. International journal of oral and maxillofacial surgery. 2011 Apr 1;40(4):353-9.

4. Bailey LJ, Collie FM, White Jr RP. Long-term soft tissue changes after orthognathic surgery. The International journal of adult orthodontics and orthognathic surgery. 1996 Jan 1;11(1):7-18.

5. Bell WH, Jacobs JD, Quefada JG. Simultaneous repositioning of the maxilla, mandible, and chin treatment planning and analysis of soft tissues. American Journal of Orthodontics. 1986 Jan 1;89(1):28-50.

6. Bo-Ram kim, Oh KM, Cevidanes LH, Park JE, Sim HS, Seo SK, Reyes M, Kim YJ, Park YH. Analysis of 3- D soft tissue changes after 1 and 2-jaw orthognathic surgery in mandibular prognathism patients. American association of oral and maxillofacial surgeons 2013;71:151-161

7. Eggensperger NM, Lieger $O$, Thüer U, Iizuka $T$. Soft tissue profile changes following mandibular advancement and setback surgery an average of 12 years postoperatively. Journal of oral and maxillofacial surgery. 2007 Nov 1;65(11):2301-10.

8. Higgins JP, Green S. Cochrane Handbook for Systematic Reviews of Interventions Version 5.1.0. Cochrane Collaboration; 2011;8:1-674.

9. Incrapera $\mathrm{AK}$, Kau $\mathrm{CH}$, English JD, McGrory K, Sarver DM. Soft tissue images from cephalograms compared with those from a 3D surface acquisition system. The Angle Orthodontist. 2010 Jan;80(1):5864.

10. Junho Jung, Chi-Heun Lee, Jung-Woo Lee, and Byung-Joon Choi. Three dimensional evaluation of soft tissue after orthognathic surgery. Head Face Med. 2018; 14: 21.

11. Khanehmasjedi M, Miresmaili A, Jafari S, Khanehmasjedi S. Comparison of the soft tissue orthodontic analysis measurements between conventional lateral cephalograms and CBCT derived lateral cephalograms.2017. 
12. Kyung-A Kim, Ye-Jin Chang, Su-Hyun Lee, HyunJoon An and Ki-Ho Par. Three-dimensional soft tissue changes according to skeletal changes after mandibular setback surgery by using cone beam computed tomography and a structured light scanner. Progress in Orthodontics 2019; 1:20:25.

13. Landes $\mathrm{CA}$, Zachar $\mathrm{R}$, Diehl $\mathrm{T}$, Kovács AF. Introduction of a three-dimensional anthropometry of the viscerocranium. Part II: evaluating osseous and soft tissue changes following orthognathic surgery. Journal of Cranio-Maxillofacial Surgery. 2002 Feb 1;30(1):25-34

14. Liberati A, Altman DG, Tetzlaff J, Mulrow C, Gøtzsche PC, Ioannidis JP, et al. The PRISMA statement for reporting systematic reviews and meta-analyses of studies that evaluate health care interventions: explanation and elaboration. J Clin Epidemiol 2009;10:1-34.

15. Maal TJ, de Koning MJ, Plooij JM, Verhamme LM, Rangel FA, Bergé SJ, Borstlap WA. One year postoperative hard and soft tissue volumetric changes after a BSSO mandibular advancement. International journal of oral and maxillofacial surgery. 2012 Sep 1;41(9):1137-45.

16. Modig $M$, Andersson L, Wårdh I. Patients' perception of improvement after orthognathic surgery: pilot study. British Journal of Oral and Maxillofacial Surgery. 2006 Feb 1;44(1):24-7

17. M. Verdenik, N. IhanHren. Differences in three dimensional soft tissue changes after upper, lower, or both jaw orthognathic surgery in skeletal class III patients. Int. J. Oral Maxillofac Surg. 2014; 6:17.
18. Park YC, Burstone CJ. Soft-tissue profile-fallacies of hard-tissue standards in treatment planning. American Journal of Orthodontics and Dentofacial Orthopedics. 1986 Jul 1;90(1):52-62.

19. Shamseer L, Moher D, Clarke M, Ghersi D, Liberati A, Petticrew M, et al. Preferred reporting items for systematic review and meta-analysis protocols (PRISMA-P) 2015: Elaboration and explanation. BMJ 2015;349:1-25

20. Rahul Tiwari, P. Srinivas Chakravarthi. A Perioral Soft

Tissue evaluation after Orthognathic Surgery Using Three Dimensional Computed Tomography Scan. The Open Dentistry Journal, 2018, 12, 366-376

21. Rustemeyer J, Gregersen J. Quality of life in orthognathic surgery patients: post-surgical

improvements in aesthetics and self-confidence. Journal of cranio-maxillofacial surgery. $2012 \mathrm{Jul}$ 1;40(5):400-4.

22. Ryckman MS, Harrison S, Oliver D, Sander C, Boryor AA, Hohmann AA, Kilic F, Kim KB. Soft-tissue changes after maxillomandibular advancement surgery assessed with cone-beam computed tomography. American Journal of Orthodontics and Dentofacial Orthopedics. 2010 Apr 1;137(4):S86-93.

23. Yong-Kyu Lim; Eun-Hye Chu; Dong-Yul Lee; IlHyung Yang; Seung-HakBaek. Three-dimensional evaluation of soft tissue change gradients after mandibular setback surgery in skeletal Class III malocclusion. Angle Orthodontist, 2010;5:896-903 